\title{
The enhancement of schedule-induced polydipsia by FR-20 and FR-80 lick-contingent shock
}

\author{
GLEN D. KING \\ Auburn University, Auburn, Alabama 36830
}

\begin{abstract}
Seven food-deprived female albino rats with electrode attachments to the backs of their necks were divided into two groups, a FR-20 lick-contingent-shock group (four subjects) and a FR-80 lick-contingent-shock group (three subjects). The subjects were exposed to five successive experimental phases, including a baseline phase during which 100 food pellets and water were freely available; a FT 1-min reinforcement schedule phase during which the 100 pellets were delivered intermittently; a FR lick-contingent-shock phase which was the same as the reinforcement schedule phase with the addition that animals were given a $.1-\mathrm{mA} .5$-sec shock for the first 20 or 80 postpellet licks in the interpellet interval; a replication of Phase 2 and a replication of Phase 1 . Water intake under intermittent schedules of reinforcement increased when specifically "punished" with the mild electric shock for both groups, with the FR-80 group showing larger increments than the FR-20 group. Increments in water intake associated with shock presentation appeared to be due to an increase in lick-burst length and frequency of initiation of drinking.
\end{abstract}

Segal and Oden (1965) proposed that excessive drinking in schedule-induced polydipsia (SIP) develops as a result of any one or a combination of determinants, including timing behaviors, adventitious reinforcement, a thirst state, and "emotional pacification," which may be akin to the anxiety assumed to underlie compulsive eating or drinking in humans. A great deal of research has been devoted to assessing the credibility of the first three of these possible explanations for SIP, but relatively little experimental investigation has been devoted to determining the role of emotional factors in the initiation and maintenance of SIP.

Although it may be difficult at first to accept the notion that excessive water intake may develop in organisms as a result of emotional arousal, various experiments have provided strong evidence that this may be the case. It appears that excessive drinking occurs, or is substantially enhanced, by raising monkeys in total isolation (Miller, Mirsky, Caul, \& Sakata, 1969), exposing rats to a strange environment with or without shock (Moyer, 1965), applying mild noncontingent footshock to polydipsic rats (Segal \& Oden, 1969), or exposing rats to a scheduled delivery of $1002-\mathrm{mA}$ electric shocks before the SIP paradigm is introduced (King, 1974).

An explanation of these experimental findings may be that when an organism is exposed to conditions such as food deprivation, intermittent schedules of reinforcement, and other potentially aversive stimuli, an

This research was supported in part by Public Health Service Research Grants MH-08755 and MH-12025, Robert W. Schaeffer, principal investigator, and the Auburn University Research Council. The experiment reported comprised part of the author's doctoral dissertation at Florida State University. Robert W. Schaeffer sponsors this paper and takes full editorial responsibility for its content. Requests for reprints should be submitted to Glen D. King at the Department of Psychology. emotional state develops in the organism which produces an energization of the most highly probable response. In SIP, excessive drinking occurs because, when the rat consumes food, it is highly probable that drinking will follow, and this is the response which is energized. If SIP is an energization of the normal drinking response following food reinforcement due to some emotional state, the amount of drinking, as the indicator of the intensity of that emotional state, should be subject to change by altering the emotional state. This theoretical notion proposes that stimuli normally considered as "punishing" may in fact energize emotional behaviors after which they occur, if the suggested punishment imposes a low enough level of stress such that the energization factor is predominant over the disruptive factor.

This experiment was designed to demonstrate that schedule-induced drinking, which is presumed to be an emotional behavior, is further augmented by an aversive stimulus, electric shock. In this experiment, low-level shock was made specifically contingent upon a fixed number of postpellet lick responses. By shocking the subjects during the postpellet lick burst (as opposed to Segal \& Oden's noncontingent shock procedure, 1969), drinking would be expected to increase if the contingent electric shock is at a low enough level such that the energization (i.e., motivational or arousal) gradient of punishment is predominant over the aversive or disruptive gradient. These results were anticipated, since an experiment reported by Ulrich and Craine (1964) demonstrated that shock-elicited aggression, when punished by electric shock, increased rather than decreased.

The relatively low intensity of shock $(.1 \mathrm{~mA})$ was chosen to ensure that the level of stress caused by the electric stimulation was not extremely disruptive. In this respect, Cohen (1968) has provided evidence that a 
shock of $.1-\mathrm{mA}$ intensity is punishing in that latencies to pressing a bar for food, an operant which was punished with a $.1-\mathrm{mA}$ shock, increased relative to a no-shock barpressing condition. However, this level of shock is not intense enough to elicit competing responses, or cause debilitation of the animal. The results predicted from the proposed theory are that behaviors such as schedule-induced polydipsia, when followed by a mild $.1-\mathrm{mA}$ shock, will increase.

\section{METHOD}

\section{Subjects}

The subjects were eight naive female albino Dublin SDD/DR rats, approximately 120-150 days old. The animals were individually housed under conditions of constant illumination, temperature, and humidity. During the experiment, the subjects were maintained at $80 \%$ of their free-feeding weight, with water freely available both in the home cage and in the experimental chamber. During the experimental baseline, one subject expired. No data collected for this subject were considered.

\section{Apparatus}

Eight Lehigh Valley Electronics, Model 1316, experimental chambers were employed. In each chamber, the right lever was removed to allow for the insertion of a drinking tube spout. The end of the spout, slightly recessed from the operant panel inside the chamber, was $7 \mathrm{~cm}$ from the delivery tube in the food magazine and $3.5 \mathrm{~cm}$ from the chamber floor grids. A clarostat potentiometer, Model 7936-10, was firmly attached to the top of each Plexiglas experimental chamber. Attached to the base of each potentiometer, which extended into the experimental chamber, was a spring, approximately $14 \mathrm{~cm}$ in length, with an adjustable pin at the free end. This pin was attached to an electrode pin present in the back of the neck of each subject. Each potentiometer was modified slightly to allow the animal access to all parts of the experimental chamber while retaining electrical continuity for delivery of shock. The electrical shock was delivered from the unscrambled shock source of a Grason-Stadler shock generator, Model 36070B. Delivery of shock and Noyes food pellets and recording of shocks and responses on an Esterline Angus digital operations recorder were programmed by standard relay circuitry.

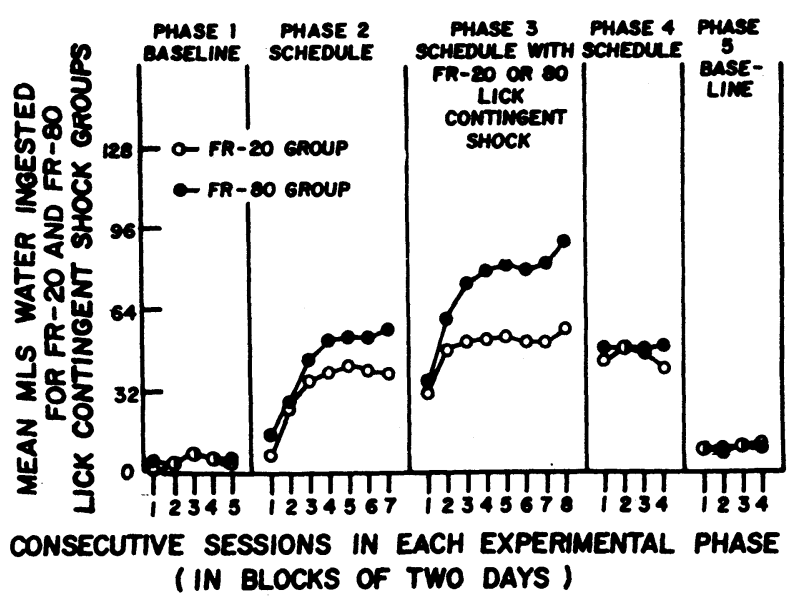

Figure 1. Mean water intakes during each experimental phase across all subjects in both the FR-20 and FR-80 lick-contingent shock groups.

\section{Procedure}

For all phases of the experiment, each subject had the spring attachment from the potentiometer connected to the pin in the nape of its neck for each entire daily session. The pins were inserted during a minor surgical operation and were replaced as necessary immediately following daily sessions.

In Phase 1 (baseline), 100 45-mg Noyes food pellets were placed in the foodcup to accomplish magazine training. The subjects remained in the test chambers for 100 min each session. Phase 1 continued until water intakes stabilized for all subjects, with stabilization for all phases defined as the condition in which the mean water intake for the last three sessions minus the mean for the preceding three sessions divided by the mean of the last six sessions did not exceed 20 .

In the second phase of the experiment, $10045-\mathrm{mg}$ food pellets were delivered on a FT 60 -sec intermittent schedule. A FT schedule is one in which food pellets are delivered on a periodic basis independently of the organism's behavior. Phase 2 continued until polydipsia developed and stabilized in all animals. Phase 3 was a replication of Phase 2 with the addition of electric shock to subjects during the postpellet lick bursts that are characteristic of schedule-induced polydipsia. The seven subjects were assigned to two groups with four subjects in one group and three subjects in the other. Groups 1 and 2 received $.1-\mathrm{mA} .5-\mathrm{sec}$ shocks after the first 20 licks in the interpellet interval and after the first 80 licks in the interpellet interval, respectively. Different postpellet lick requirements for contingent shock were chosen to note the effects of two different temporal presentations of shock on the length of the drinking response. If a subject fell short of the lick requirement necessary to receive shock in any interpellet interval, no shock was delivered in that interval; and, on delivery of the next food pellet, the lick requirement for shock was automatically reset to either 20 or 80 for respective groups.

Phases 4 and 5 were replications of Phases 2 and 1 , respectively.

\section{RESULTS AND DISCUSSION}

The mean water intakes for each group across phases of the experiment are presented in Figure 1. The mean data presented are representative of individual subjects in each group. In consideration of those subjects to which shock was delivered contingent on 20 postpellet licks, the data presented in Figure 1 indicates a slight trend to increase water intake in the shock phase relative to the preceding and following schedule phases without shock, while the subjects in the FR-80 group increased remarkably in the shock phase relative to preceding and following no-shock phases.

Analyses of variance for repeated measures indicated that, for both groups, the amounts of water ingested during the schedule conditions (Phases 2 and 4 ) were significantly greater $(p<.05)$ than those amounts ingested during baseline (Phases 1 and 5) and significantly less $(p<.05)$ than the amounts ingested during the shock phase (Phase 3 ). In addition, the FR- 80 group drank significantly more than the FR-20 group only during the lick-contingent shock condition $(p<.05)$. It appears that for both groups, the introduction of the schedule condition resulted in increased water ingestion relative to baseline and; furthermore, for both groups, the introduction of lick-contingent electric shock resulted in an additional 
increment in water ingestion relative to the schedule condition without shock.

Lick burst length was determined for each animal for the last 2 days of each condition. In the FR-20 group, a lengthening of the lick burst during the shock condition occurred for all four animals, whereas an increase in total frequency of lick bursts was obtained in only one subject. This particular FR-20 animal (Subject 105) seemed to increase both lick burst length and frequency, relative to those response measures for the schedule condition without shock in Phases 2 and 4. Similarly, FR-80 animals also seemed to increase lick burst length when subjected to the shock conditions. In addition, they were more likely to increase the frequency with which lick bursts were initiated. Analysis of the data also indicated no difference between groups in the number of food pellet deliveries followed by at least one lick burst, indicating that the higher frequency of drinking in the FR-80 group was a result of an increased number of drinking bouts within the interpellet interval.

In addition, the number of shocks earned by each subject did not differ appreciably across groups, indicating that the greater amount of drinking in FR-80 subjects compared to FR-20 subjects was not attributable to a difference in total electric shocks received. By observation, it was noted that, initially, shock interrupted drinking; but as the water intakes stabilized, the animals drank through the shock delivery, although they were noticeably still affected by the shock, as indicated by their laying their ears back, becoming suddenly tense, etc. It appears that an electric shock (which is considered to be punishing at the $.1-\mathrm{mA}$ level; Cohen, 1968), will, when its application is made contingent on the occurrence of a schedule-induced behavior, increase the intensity of that behavior. In the case of SIP, the application of a mildly aversive electric shock contingent on postpellet licking resulted in an increase in response intensity as indicated by increased water intake. This increase in water intake seems to be produced by an energization of responding along one or both of two dimensions of the drinking response, including increases in lick burst length and lick burst frequency. These results corroborate and extend the results reported by Galantowicz and King (1975) for $.1-\mathrm{mA}$ lick-contingent shock. These studies considered together appear to lend further support to the emotional explanation for SIP.

\section{REFERENCES}

Conen. P. S. Punishment: The interactive effects of delay and intensity of shock. Journal of the Experimental Analysis of Behavior. 1968, 11, 789-799.

Galantowicz. E. P., \& King, G. D. The effects of three levels of lick-contingent footshock on scheduleinduced polydipsia. Bulletin of the Psychonomic Society. 1975, 5. 113-116.

KING. G. D. The enhancement of schedule-induced polydipsia by preschedule noncontingent shock. Bulletin of the Psychonomic Society. 1974, 3, 46-48.

Miller. R. E.. Mirsky, I. A., Caul, W. F.. \& SAKATA. T. Hyperphagia and polydipsia in socially isolated rhesus monkeys. Science, 1969, 165, 1027-1028.

MoYer. K. E. Effect of experience with emotion provoking stimuli on water consumption in the rat. Psychonomic Science, 1965 , 2. $251-252$.

Segal. E. F.. \& Oden. D. L. Determinants of polydipsia in rats: A reply to Stein. I. Emptying the water bottle. Psychonomic Science. 1965. 2. 201-202.

Segal. E. F.. \& Open, D. L. Effects of drinkometer current and of footshock on psychogenic polydipsia. Psychonomic Science. 1969. 14. 13-15.

Ulrich. R. E.. \& Craine, W. H. Persistence of shock-induced aggression. Science. 1964, 143, 971-973.

(Received for publication July 10, 1975.)

\section{ERRATUM}

White, K. G., \& Silver, A. B. Cerebral hemispheres serve as two channels for visual information. Bulletin of the Psychonomic Society, 1975, 6, 51-52. The last sentence of the first paragraph on page 52 should read: "Thus, in the situation where a grid pattern was directed to one hemisphere, and a CVC was simultaneously directly to the other, identification was more accurate when spatial stimuli were processed by the right (spatial) hemisphere and verbal stimuli were processed by the left (verbal) hemisphere than when spatial and verbal stimuli were processed by "inappropriate" hemispheres." 